Damien P. Kelly, Nail Sabitov, Thomas Meinecke and Stefan Sinzinger :

Some considerations when numerically calculating diffraction patterns

Zuerst erschienen in:

Digital Holography and Three-Dimensional Imaging (DH), OSA Techinal Digest (CD) (Optical Society of America, 2011), paper DTuC5. 


\title{
Some considerations when numerically calculating diffraction patterns.
}

\author{
Damien P. Kelly*, Nail Sabitov, Thomas Meinecke, Stefan Sinzinger. \\ Department of Mechanical Engineering, \\ Institute for Micro-Nanotechnologies, \\ Technical Ilmenau University of, Technology \\ P.O. Box 100565 , \\ D-98693 Ilmenau. \\ *damien-peter.kelly@tu-ilmenau.de
}

\begin{abstract}
Numerical calculation of diffraction integrals remains a challenge in modern optics, with applications in digital holography, phase retrieval techniques and wave-front sensing. Two different numerical techniques are compared and the associated sampling rules derived.
\end{abstract}

OCIS codes: (070.2025) Discrete optical signal processing; (070.7345) Wave propagation; 090.1995 Digital holography.

\section{Introduction}

Efficient numerical techniques for calculating diffraction patterns are important in modern optics from studying fundamental diffraction problems to reconstructing digital holograms in close to real time. The Fast Fourier Transform (FFT) is often the basis for these algorithms, however its use often obscures fundamental sampling issues that arise when implementing the numerical routines. The purpose of this manuscript is to briefly outline some fundamental properties that should be considered when implementing these algorithms. We begin by considering a standard Fresnel based digital holographic (DH) system as depicted in Fig. 1. For simplicity we perform a 1-D analysis. A unit amplitude plane wave is incident on a transmittance function, $U(X)$, and the diffracted light propagates to the CCD plane where the optical field is sampled at discrete and regularly spaced intervals. We assume that some processing (for example phase shifting interferometry techniques [1]) is performed on the captured hologram and that a discrete recording of the complex amplitude at the CCD plane, $u(x)$, is returned by the camera. In order to concentrate on the numerical issues that arise when reconstructing the hologram, we ignore important image performance limiting factors such as the finite pixel size and the finite extent of the camera referring the reader instead to Ref. [2] for more detail.

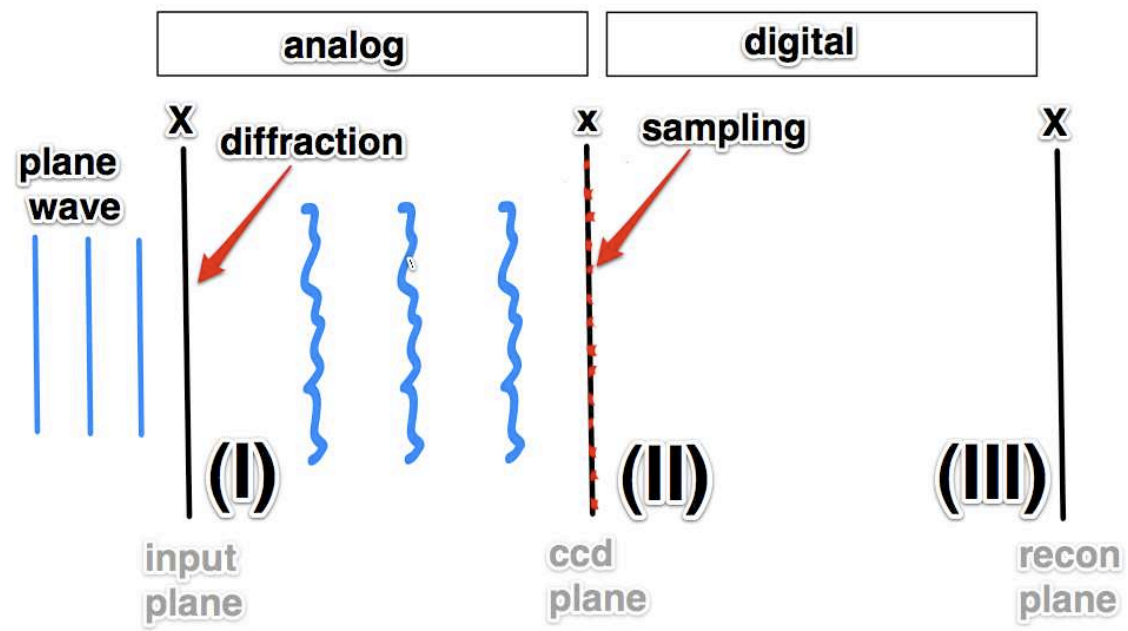

Fig. 1 DH imaging system comprising (i) Propagation of a diffracted optical wavefield, (ii) Discrete recording of complex field and (iii) Numerical reconstruction of hologram.

We assume that our input field, $U(X)$, is given by,

$$
U(X)=\operatorname{rect}(X) \cos \left(2 \pi f_{x} X\right)
$$

where $\operatorname{rect}(X)=1$, when $|X|<L / 2$ and 0 otherwise. Eq. (1) acts to limit the power entering the optical system to $L / 2$ and ensures a finite spatial extent such that $-L / 2<X<L / 2$. By varying $L$ we can control the extent of our input field and by varying $f_{\mathrm{x}}$ we can control the spatial frequency content of our signal. We can relate $u(x)$ to $U(X)$ using an inverse Fresnel transform, 


$$
U(X)=\frac{1}{\sqrt{-j \lambda z}} \int_{-\infty}^{\infty} u(x) \exp \left[\frac{-j \pi}{\lambda z}(X-x)^{2}\right] d x
$$

or equivalently,

$$
U(X)=F^{-1}\left\{\hat{u}(f) \exp \left(j \pi \lambda z f^{2}\right)\right\}(X),
$$

where $F$ and $F^{-1}$ refer to forward and inverse Fourier Transform (FT) operations [see Eq. (2.1) in [3]], and where $\hat{u}(f)=F\{u(X)\}(f)$. The variable $f$ indicates that we are in the spatial frequency domain. Eq. (2 a) and (2 b) are mathematically equivalent and form the basis for the implementing the Direct Method (DM) and Spectral Method (SM) respectively [4], which are fast discrete numerical techniques for calculating the Fresnel diffraction integral. There are however subtle but important differences between these two numerical implementations that we shall address in Section 2. Once $u(x)$ is sampled at a discrete set of spatial locations, $\mathrm{x}_{\mathrm{D}}$ $=\left[x_{1}, x_{2}, \ldots, x_{\mathrm{N}}\right]$, an $N$ dimensional array of complex numbers is returned which we write as,

$$
\begin{aligned}
\mathrm{u}_{\mathrm{D}} & =u(x) \delta_{\mathrm{T}}(x) \\
& =\left[u_{1}, u_{2}, \ldots . ., u_{n}\right]
\end{aligned}
$$

where

$$
\delta_{\mathrm{T}}(X)=\sum_{n=1}^{N} \delta(x-n T),
$$

and $\delta(x)$ is the Dirac delta function. The variable $T$ is the distance between the centers of adjacent pixels on the camera. We now examine how to reconstruct the continuous field, $R^{(\mathrm{S} / \mathrm{D}) \mathrm{M}}(X)$, from a discrete set of samples.

\section{Numerical analysis}

In this section we examine some theoretical aspects that arise when numerically calculating a paraxial regime diffraction pattern. In the literature two different fast numerical techniques are commonly used, i.e. the DM and SM based on Eq. (2 a) and (2 b) respectively. The first approach uses a single FFT operation while the second requires two FFT operations [4]. The SM approach however can be extended so that the more accurate RaleighSommerfeld diffraction integrals may be calculated and hence may be important for microscopy applications [5].

\subsection{Direct Method}

Using Eq. (2 a) and (3) we can write the reconstructed field as

$$
R^{\mathrm{DM}}(X)=K_{1} \sum_{n=1}^{N} u_{n} \exp \left[\frac{-j \pi}{\lambda z}\left(X-x_{n}\right)^{2}\right]
$$

where $K_{1}$ is constant chosen to normalize $\left|\mathrm{R}^{\mathrm{DM}}(X)\right|$. We note that although two discrete arrays, $\mathrm{u}_{\mathrm{D}}$ and $\mathrm{x}_{\mathrm{D}}$, are used in the calculation, the output variable $X$ is continuous. The effect of using an FFT to implement this calculation is to restrict the output variable $X$ to specific values, see Eq. (5) and (10) in Ref. [4]. It is important to note that this constraint is imposed by the nature of the FFT algorithm therefore in principal there is no restriction on the value of $X$. An analytical form for $u(x)$, exists [6], that we sample at intervals of $T \sim 0.03738$ $\mathrm{m}$, resulting in a Nyquist frequency of $f_{\mathrm{NQ}}=1 /(2 T)=13.375 \mathrm{~m}^{-1}$.
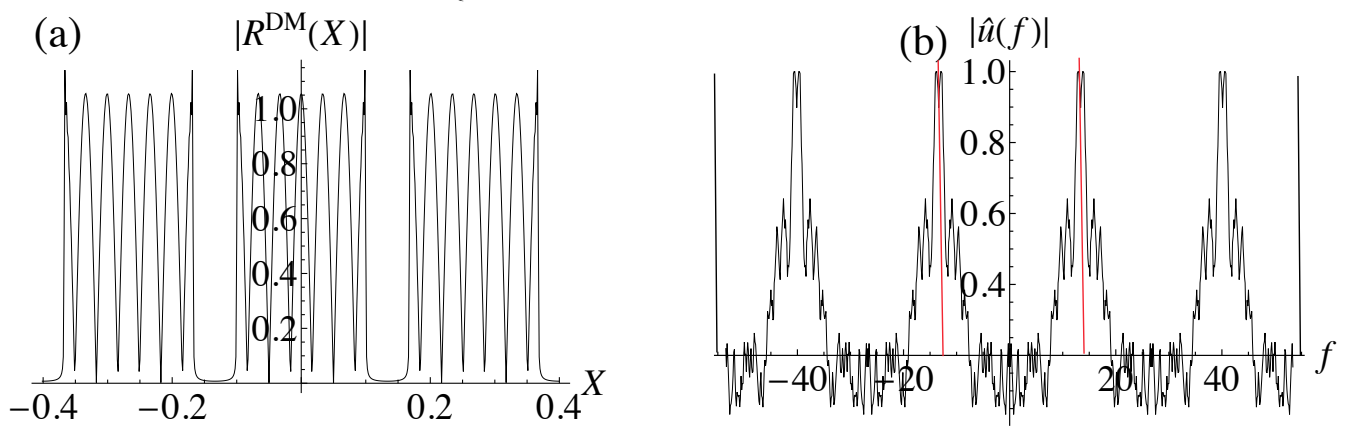

Fig. 2. (a) Plot of $\left|R^{\mathrm{DM}}(X)\right|$ for values indicated in the text. The replicas arise due to the sampling process at the camera. (b) Plot of $\mathrm{u}$ again showing replicas. This is the first step for implementing the SM. The two red lines and black lines indicate the extent of the space frequency domain that is allowed to contribute to the $\left|\mathrm{R}^{\mathrm{SM}}(X)\right|$ in Fig. 3(a) and (b) respectively. 


\section{DTuC5.pdf}

In Fig. 2(a) we present a numerically calculated reconstruction with $z=10 \mathrm{~m}, \lambda=0.001 \mathrm{~m}, N=214, f_{\mathrm{x}}=15 \mathrm{~m}^{-1}$ and $L=0.2$. We note that although we have sampled at a rate below the Nyquist, i.e. $f_{\mathrm{x}}>f_{\mathrm{NQ}}$, we can still recover our input signal. Replicas are clearly visible and separated from each other by a distance $\lambda z / T=0.6275$ $>L$ indicating that we can perfectly recover our input signal [2].

\subsection{Spectral Method}

We now attempt to reconstruct our input signal using the SM. In contrast to the DM approach, this is a two-step procedure where a FT is first performed on $\mathrm{u}_{\mathrm{D}}$ resulting in the continuous signal,

$$
\hat{u}(f)=K_{2} \sum_{n=1}^{N} u_{n} \exp \left(-j 2 \pi f x_{n}\right),
$$

where $K_{2}$ is a normalizing constant. Using the same values as previously we plot Eq. (6), and present the result in Fig. 2(b). We note that the signal is strongly aliased since $u(x)$ has been sampled at a sub-Nyquist rate. The replicas in the FT domain are separated from each other by $1 / T$. To complete the reconstruction, $\hat{u}(f)$ is multiplied by a chirp function $\left[\exp \left(\mathrm{j} \pi \lambda z f^{2}\right)\right.$, see Eq. (2)], before an inverse FT operation on the product is performed, to yield $R^{\mathrm{SM}}(X)$. Several questions now arise when implementing the inverse FT integral: (i) What are appropriate integration limits, $f=+/-\Delta f / 2$ ? (ii) What happens if we use only a finite number of samples, $N_{\mathrm{f}}$, to calculate the inverse operation numerically? In order to investigate question (i), we limit the region of integration $-1 /(2 T)<f<1 /(2 T)$ [as indicated by the red lines in Fig. 2(b)] and examine the reconstructed field which is plotted in Fig. 3(a).
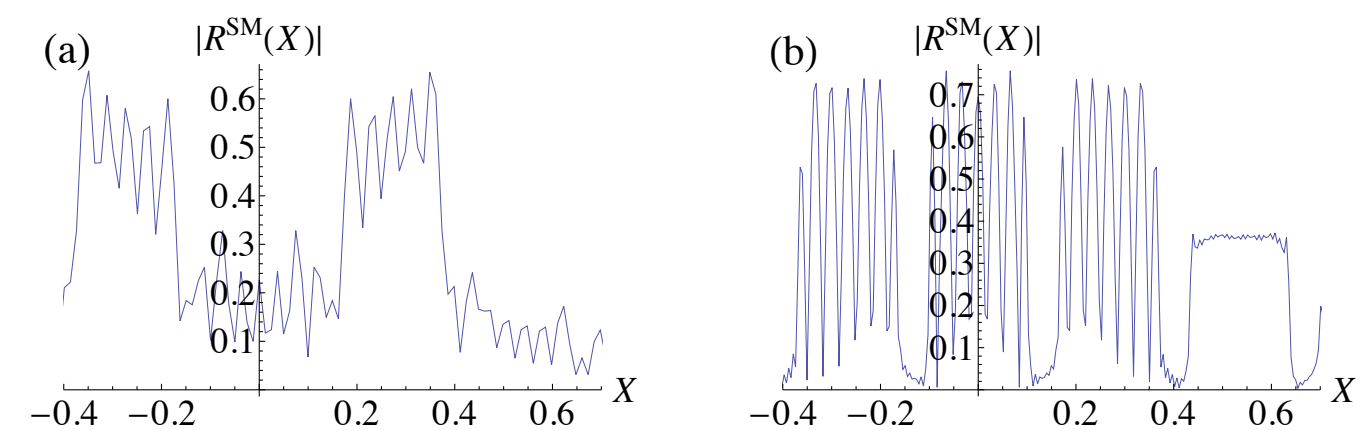

Fig. 3 Plot of $\left|R^{\mathrm{SM}}(X)\right|$ when two different aliased spatial frequency extents are allowed to contribute to the calculation, see Fig. 2.

There is a striking difference between the distributions in Fig. 2(a) and Fig. 3(a), which we suspect could be attributed to (a) the aliased nature of $\hat{u}(f)$ or (b) the integration limits. To calculate the result presented in Fig. 3(b), we increase $\Delta f$ to $4 / \mathrm{T}$ allowing higher order spatial frequency replicas [the black lines in Fig. 2(b) indicate the integration limits] to contribute to the inverse FT. In this instance we note that the result is closer to that in Fig. 2(a). Indeed in our simulations we have found that as $\Delta f$ increases the $R^{\mathrm{SM}}(X)$ and the $R^{\mathrm{DM}}(X)$ solutions appear to converge. We note that if $\Delta f$ is finite, it acts as a low pass filter and convolves the $R^{\mathrm{SM}}(X)$ with a sinc function, distorting the reconstruction significantly. This is evident when one compares Fig. 2 and 3. If we wish to recover spatial frequencies above the Nyquist limit using the SM algorithm we must include contributions from higher order replicas in the spatial frequency domain. The spatial frequency extent at the output of an FFT algorithm can be controlled using the technique described in Appendix B of Ref. [2].

We now turn to point (ii) raised above, if we sample the function, $\hat{u}(f)$, in steps of $\delta \mathrm{f}=\Delta f / N_{\mathrm{f}}$, then we will generate a new set of replicas in the space domain separated from each other by a distance $1 / \delta$ f. This second set of replicas, occur because we are effectively sampling on two separate occasions when we implement the SM calculation, once at the camera plane, and again in the Fourier plane. While the sampling rate at the camera plane is determined by the CCD camera, we can chose how we wish to sample the Fourier plane distribution.

\section{References}

1. I. Yamaguchi and T. Zhang, "Phase-shifting digital holography", Opt. Lett. 2, 1268-1270 (1997).

2. D. P. Kelly, B. M. Hennelly, N. Pandey, T. J. Naughton, and W. T. Rhodes, "Resolution limits in practical digital holographic systems", Optical Engineering 48, 095801 (2009).

3. J. Goodman, Introduction to Fourier Optics, 2nd ed. (McGraw-Hill, New York, 1966).

4. D. Mas, J. Garcia, C. Ferreira, L. M. Bernardo, and F. Marinho, "Fast algorithms for free-space diffraction patterns calculation," Opt. Commun. 164, 233-245 (1999).

5. N. Delen and B. Hooker, "Free-space beam propagation between arbitrarily oriented planes based on full diffraction theory: a fast Fourier transform approach," J. Opt. Soc. Am A, 15, 857-867 (1998).

6. D. P. Kelly, B. M. Hennelly, C. McElhinney, and T. J. Naughton, "A practical guide to digital holography and generalized sampling,"Proc. SPIE 7072, 707215 (2008). 